\title{
Cytokine and microRNA levels during different periods of paradoxical sleep deprivation and sleep recovery in rats
}

\author{
Malinalli Brianza-Padilla ${ }^{1}$, Fausto Sanchez-Muñoz ${ }^{2}$, Gonzalo Vazquez-Palacios ${ }^{3}$, Fengyang Huang ${ }^{4}$, Julio \\ Cesar Almanza-Perez ${ }^{5}$, Rafael Bojalil ${ }^{\text {Corresp., }}{ }^{2,6}$, Herlinda Bonilla-Jaime $^{\text {Corresp. } 7}$ \\ 1 Posgrado en Biologia Experimental, División de Ciencias Biológicas y de la Salud, Universidad Autonoma Metropolitana Iztapalapa, Ciudad de Mexico, \\ Mexico \\ 2 Departamento de Inmunología, Instituto Nacional de Cardiologia Ignacio Chavez, Ciudad de Mexico, Mexico \\ 3 Colegio de Ciencias y Humanidades, Universidad Autonoma de la Ciudad de Mexico, Ciudad de Mexico, Mexico \\ 4 Laboratorio de Investigación en Farmacología y Toxicología, Hospital Infantil de Mexico Federico Gomez, Ciudad de Mexico, Mexico \\ 5 Departamento de Ciencias de la Salud, División de Ciencias Biológicas y de la Salud, Universidad Autonoma Metropolitana Iztapalapa, Ciudad de Mexico, \\ Mexico \\ 6 Departamento de Atención a la Salud, Universidad Autónoma Metropolitana Xochimilco, Ciudad de México, México \\ 7 Departamento de Biologia de la Reproducción, División de Ciencias Biológicas y de la Salud, Universidad Autonoma Metropolitana Iztapalapa, Ciudad de \\ Mexico, Mexico \\ Corresponding Authors: Rafael Bojalil, Herlinda Bonilla-Jaime \\ Email address: rafaelbojalil@gmail.com, bjh@xanum.uam.mx
}

\begin{abstract}
Background. Sleep has a fundamental role in the regulation of homeostasis. The aim of this study was to assess the effect of different periods of paradoxical sleep deprivation (PSD) and recovery on serum levels of cytokines and miRNAs related to inflammatory responses. Methods. Male Wistar rats were submitted to a PSD of 24, 96, or 192 hours, or of 192h followed by 20 days of recovery (192h PSD+R). The concentrations of corticosterone, cytokines (IL-6, TNF, IL-10, Adiponectin) and miRNAs (miR-146a, miR-155, miR-223, miR-16, miR-126, miR-21) in serum were evaluated. Results. At PSD 24h a significant increase of IL-6 and decrease of IL-10 were observed. At PSD 96h adiponectin increased. At 192h of PSD IL-6 increased significantly again, accompanied by a threefold increase of IL-10 and an increase of serum corticosterone. After 20 days of recovery (192h PSD+R) corticosterone, IL-6 and TNF levels increased significantly, while IL-10 decreased also significantly. Regarding the miRNAs at 24h of PSD serum miR146a, miR-155, miR-223, and miR-16 levels all increased. At 96h of PSD miR-223 decreased. At 192h of PSD decreases in miR-16 and miR-126 were observed. After recovery serum miR-21 increased and miR16 decreased. Conclusion. PSD induces a dynamic response likely reflecting the induced cellular stress and manifested as variating hormonal and inflammatory responses. Sleep deprivation disturbed corticosterone, cytokine and miRNA levels in serum related to the duration of sleep deprivation, as shortterm PSD produced effects similar to those of an acute inflammatory response and long-term PSD induced long-lasting disturbances of biological mediators.
\end{abstract}


1 Cytokine and microRNA levels during different periods of 2 paradoxical sleep deprivation and sleep recovery in rats.

3

4

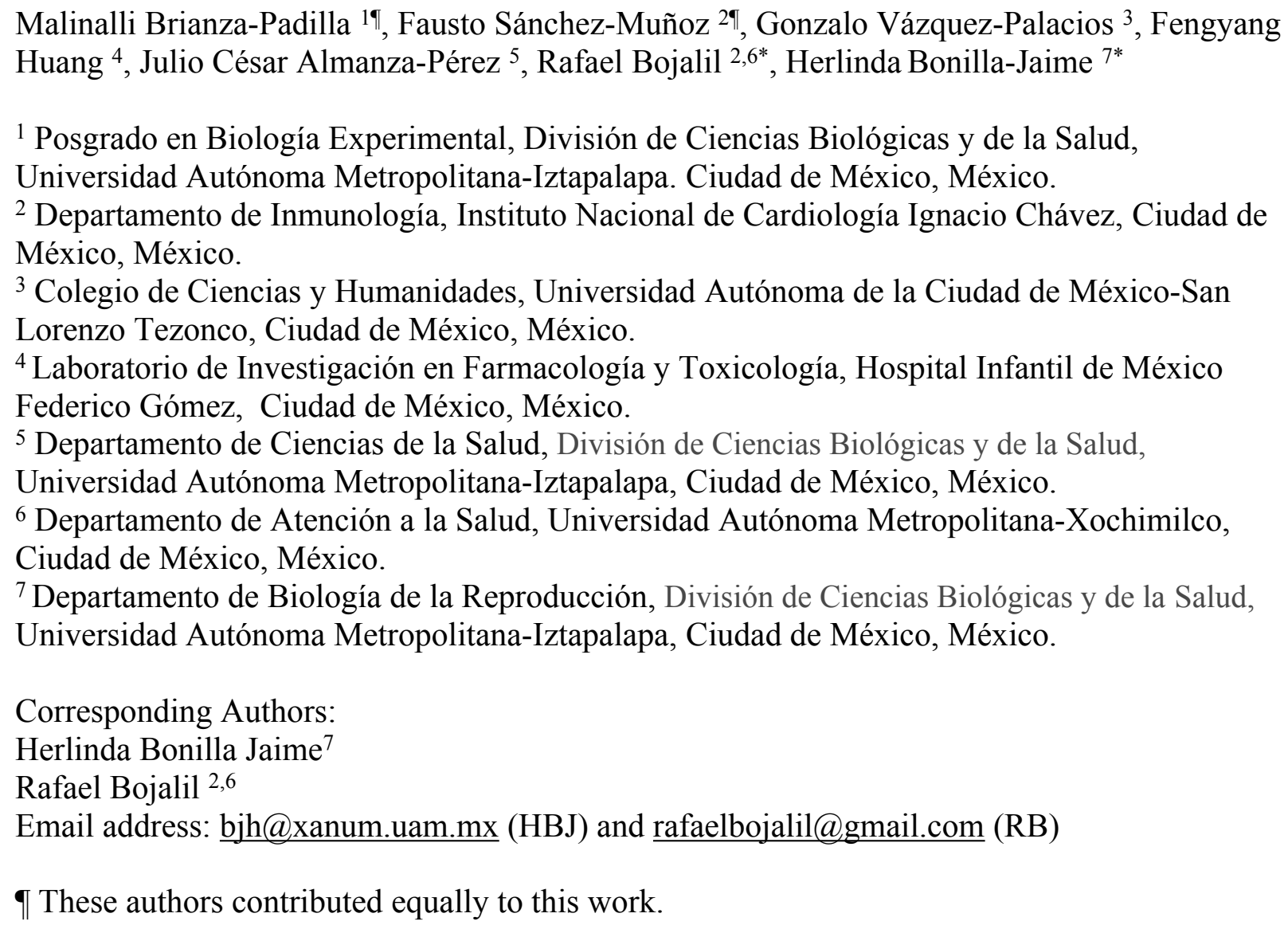

\section{Abstract}

Background. Sleep has a fundamental role in the regulation of homeostasis. The aim of this study was to assess the effect of different periods of paradoxical sleep deprivation (PSD) and recovery on serum levels of cytokines and miRNAs related to inflammatory responses.

Methods. Male Wistar rats were submitted to a PSD of 24, 96, or 192 hours, or of 192h followed by 20 days of recovery (192h PSD+R). The concentrations of corticosterone, cytokines (IL-6, 
37 TNF, IL-10, Adiponectin) and miRNAs (miR-146a, miR-155, miR-223, miR-16, miR-126, miR38 21) in serum were evaluated.

39 Results. At PSD 24h a significant increase of IL-6 and decrease of IL-10 were observed. At PSD $4096 \mathrm{~h}$ adiponectin increased. At 192h of PSD IL-6 increased significantly again, accompanied by a 41 threefold increase of IL-10 and an increase of serum corticosterone. After 20 days of recovery 42 (192h PSD+R) corticosterone, IL-6 and TNF levels increased significantly, while IL-10 decreased 43 also significantly. Regarding the miRNAs at 24h of PSD serum miR-146a, miR-155, miR-223, 44 and miR-16 levels all increased. At 96h of PSD miR-223 decreased. At 192h of PSD decreases in 45 miR-16 and miR-126 were observed. After recovery serum miR-21 increased and miR-16 decreased.

Conclusion. PSD induces a dynamic response likely reflecting the induced cellular stress and 48 manifested as variating hormonal and inflammatory responses. Sleep deprivation disturbed corticosterone, cytokine and miRNA levels in serum related to the duration of sleep deprivation, as short-term PSD produced effects similar to those of an acute inflammatory response and longterm PSD induced long-lasting disturbances of biological mediators.

\section{New \& Noteworthy}

This is the first study to show that paradoxical sleep deprivation induces distinctive changes in circulating microRNAs related to inflammation and that after 20 days of recovery from $192 \mathrm{~h}$ of paradoxical sleep deprivation the inflammatory response is not fully restored.

\section{Introduction}

Sleep is a natural, reversible physiological state characterized by a reduction in the response to external stimuli (Jones, 1998). Numerous hypotheses have been posited to explain the function of sleep, but most authors consider it a homeostatic process involved in energy conservation, in nervous system recuperation, thermoregulation, and the immune function (Siegel, 2005) Sleep

62 deprivation constitutes a relevant health problem in modern society (Tobaldini et al., 2017).

63 Research on sleep has paid special attention to the rapid eye-movement (REM) phase or 64 paradoxical sleep because deprivation of this sleep stage has been shown to induce various effects 
65 in the brain, some of which are so significant that they can induce morphological changes, that 66 include reduced proliferation of neuronal precursors in the adult hippocampus (Sahu et al., 2013), 67 and modifications of the neurochemical, hormonal and inflammatory media (James M. Krueger, 68 Frank, Wisor, \& Roy, 2016).

69 Both, at systemic and cerebral levels cytokines participate as mediators of various complex physiological processes, including sleep regulation (Lorton et al., 2006). Depending on the amount and/or duration of the loss, sleep deprivation produces multiple physiological effects, including elevations in plasma cortisol or corticosterone levels (Deborah Suchecki, Tiba, \& Tufik, 2002), suggesting that PSD induces a stress response (McEwen, 2006). PSD also induces alterations of inflammatory markers such as IL-1 $\beta$, IL-6, IL-17 and TNF (Irwin, 2006), excessive daytime secretion of IL-6 (Vgontzas et al., 1999), and modifications in coagulation and cell adhesion molecules in humans (Grandner, Sands-Lincoln, Pak, \& Garland, 2013) and in animal models (Yehuda, Sredni, Carasso, \& Kenigsbuch-Sredni, 2009). Inflammation itself can be regulated for example by adiponectin, which induces a reduction in the secretion of the cytokines IL-6 and IL8 (Dietze-Schroeder, Sell, Uhlig, Koenen, \& Eckel, 2005), and by MicroRNAs (miRNAs). Indeed, some miRNAs play a significant role in sleep regulation and may be expressed at distinct moments of sleep and in different brain structures, including cortical areas that regulate sleep duration (Davis, Clinton, \& Krueger, 2012). One recent study demonstrated that some plasmatic miRNAs can be altered as part of the physiopathology of central hypersomnia (Holm et al., 2014), obstructive sleep apnea (K. Li, Wei, Qin, \& Wei, 2017) and sleep deprivation (Matos et al., 2014). Six miRNAs (miR-146a, -155, -223, -16, -126 and -21) have been described as potential biomarkers for at least nine non-neoplastic diseases that are associated with important inflammatory processes (Haider et al., 2014). However, to the best of our knowledge, no studies have yet related different periods of PSD and recovery from it to circulating levels of these inflammation related miRNAs.

Considering that PSD is, in itself, a stressor and that there is scarce information about the effects of different periods of PSD on inflammation related mediators, and the duration of these alterations, the objectives of the present study were: 1) to determine the effects of different periods of PSD (24h, 96h, and 192h) on serum levels of: a) corticosterone; b) IL-6, TNF, IL-10, and adiponectin; and c) miRNAs related to the inflammatory response: miR-146a, -155, -233, -16, - 
95126 and -21; and 2) if a long-lasting PSD (192h) has long-term homeostatic consequences, as

96 determined by the same mediators, considering a recovery period of 20 days.

\section{Method}

98 The study utilized 3-month-old male Wistar rats weighing approximately 250 gr that were raised

99 in the animal facilities at Universidad Autónoma Metropolitana -Iztapalapa (UAMI) under the 100 conditions stipulated in the official Mexican norm (NOM-062-ZOO-1999, 2001 revision). The

101 experimental protocol was approved by the Ethics Committee of UAMI (Session 1514, verified 3-

102 15-14-2010-2018). The rats were deprived of paradoxical sleep for 24h (PSD 24h), 96h (PSD 96h),

103 and 192h (PSD 192h); a fourth group was also deprived for 192h, but measurements were done

104 after 20 days of recovery (192h PSD+R). These intervals were chosen because many prior studies

105 of sleep deprivation also used a 96h evaluation period (Machado et al., 2004, Andersen et al., 2005,

106 Machado, Tufik, \& Suchecki, 2010), but we decided to observe two additional, extreme periods

107 (24 and 192h) for comparative purposes. The rationale for adding a prolonged recovery period is

108 that this interval may allow the body to recover from acute PSD-induced alterations experienced

109 during $192 \mathrm{~h}$. Given the aims of our study, we considered the 20-day period to acknowledge long-

110 term consequences of long-lasting PSD.

111 Each group consisted of 5 rats. The control group was housed in normal boxes in the same space

112 at the animals facility. All rats were kept under a light-dark cycle (9am-9pm) at a temperature of

113 approximately $24^{\circ} \mathrm{C}$. The method of euthanasia by decapitation is approved by NOM-062-ZOO-

1141991.

\section{Paradoxical sleep deprivation Method}

116 The modified multi-platform method was used to induce PSD (D Suchecki \& Tufik, 2000). It

117 involves placing the rats in an acrylic box (127 cm long x $44 \mathrm{~cm}$ wide x $45 \mathrm{~cm}$ high) with a base

118 that has 14 platforms measuring $6.5 \mathrm{~cm}$ in diameter $\mathrm{x} 3 \mathrm{~cm}$ high with $10-\mathrm{cm}$ separations. Water is

119 added to the base of the platform to a depth of approximately $1 \mathrm{~cm}$. This method is based on the

120 loss of muscle tone that characterizes the REM sleep condition. When an experimental animal falls

121 into the water, it experiences a sudden loss of the sleep cycle. This REM sleep deprivation method

122 has been studied previously and is considered reproducible (Machado et al., 2004). Studies have 
123 demonstrated that using this method of inducing PSD, animals not only lose REM sleep entirely,

124 but also approximately 37\% of slow wave sleep (SWS) (Machado et al., 2004).

125 Although the modified multi-platform method, has been widely used and endorsed for PSD 96h

126 (Suchecki \& Tufik, 2000, Machado et al., 2004), there is no information regarding the effect of

127 this method with other periods of PSD on loss of REM sleep and non-REM sleep. Thus, in order

128 to acknowledge the brain activity in each of the studied periods of PSD, polysomnography is

129 warranted. Another handicap is that recovery was not studied for shorter periods of PSD.

130 Quantification of serum corticosterone and cytokines levels

131 To evaluate corticosterone and cytokine concentrations, sera were obtained by decapitation,

132 ensuring that euthanasia was always carried out at the exact same hour: 9:00 am. Blood was drawn

133 in vacutainer tubes with separation gel, serum samples were aliquoted and preserved at $-70^{\circ} \mathrm{C}$ until

134 they were used. To measure corticosterone concentrations, an ELISA Test Kit Mouse/Rat was

135 employed (Alpco, Salem, NH) following the manufacturer's instructions. (sensitivity 6.1ng/ml).

136 To measure cytokine concentrations, determinations were conducted also by ELISA: For IL-6 we

137 used Prepro Tech (Rocky Hill /NJ) (range 62- 8000pg/mL), for IL-10 and TNF we used Thermo

138 Fisher Scientific kits (Rockford, IL, U.S.A.) with sensitivity $<3 \mathrm{pg} / \mathrm{ml}$, and $11.0 \mathrm{pg} / \mathrm{ml}$ respectively;

139 and with an Alpco kit (Salem, NH) for adiponectin, with sensitivity of $=0.08 \mathrm{ng} / \mathrm{ml}$.

\section{Serum miRNA quantification}

141 RNA extraction was performed using the miRNeasy Serum/Plasma kit (Qiagen). The miRNAs 142 were reverse-transcribed with the TaqMan MicroRNA Reverse Transcription Kit (Applied 143 Biosystems, Thermo Fisher Scientific, U.S.A). The RT reaction was incubated for 30 minutes at $14416^{\circ} \mathrm{C}, 30$ minutes at $42^{\circ} \mathrm{C}$, and 5 minutes at $85^{\circ} \mathrm{C}$. The miRNAs were then amplified using a 145 TaqMan MicroRNA RT kit (Applied Biosystems, Thermo Fisher Scientific, U.S.A), and detected 146 with TaqMan TM MicroRNA Assays hsa/mus miR-146a, miR-155, miR-233, miR-16, miR-126 147 and miR-21 primers and probes (Applied Biosystems, Thermo Fisher Scientific, U.S.A). The 148 cycling conditions were: initial denaturation at $95^{\circ} \mathrm{C}$ for $10 \mathrm{~min}$, followed by 45 cycles at $95^{\circ} \mathrm{C}$ for $14915 \mathrm{~s}$, at $60^{\circ} \mathrm{C}$ for $40 \mathrm{~s}$, and at $72^{\circ} \mathrm{C}$ for $10 \mathrm{~s}$. PCR was performed with a LightCycler TM 480 II 150 System (Roche Applied Science, Basel, Switzerland) and the LightCycler 480 Probes Master kit 151 (Roche Applied Science). miRNA concentrations were normalized with the concentrations of 
152 miR-39. The miRNeasy serum/plasma Spike-In Control was added to samples after the addition 153 of QIAzol lysis reagent $\left(2 \mu \mathrm{L}\right.$ of $1.6 \times 10^{8}$ copy/ $\mu \mathrm{L}$ of cel-miR-39), which can control for varying 154 RNA isolation yields and amplification efficiency. $\Delta \mathrm{Ct}$ values were calculated with problem $\mathrm{Ct}$ 155 i.e., the reference $\mathrm{Ct}$ at which miR-39 is taken as the reference gene, represented as $2^{-\Delta \mathrm{Ct}}$ (Dehoux, 156 van Beneden, Fernández-Celemín, Lause, \& Thissen, 2003).

Statistical analysis

159 All serum cytokine concentrations and serum miRNA levels were analyzed by a one-way 160 ANOVA, followed by a post hoc Tukey-Kramer test. NCSS software was used for these analyses. 161 Statistical significance in relation to the control group and between groups was set at $\mathrm{p}<0.05$

\section{Results}

163 Corticosterone concentrations increased after long periods of PSD and stayed elevated after 164 recovery.

165 In order to assess the effects of increasing lengths of sleep deprivation on the stress response, 166 corticosterone levels were measured. Short periods of PSD had no significant effect upon serum 167 corticosterone, but it was found sharply elevated after PSD 192h as compared to the control group $168[\mathrm{~F}(4,25)=17.18, \mathrm{p}<0.0001]$. After recovery $(192 \mathrm{~h}$ PSD+R) the levels of corticosterone were 169 significantly lower than after PSD 192h but were still significantly higher than those found in 170 controls (Figure 1).

171 Circulating cytokines in rats were modified by deprivation of paradoxical sleep for different 172 periods and were maintained altered after recovery.

173 The effects of different periods of sleep deprivation on serum pro- and anti-inflammatory cytokines 174 levels were measured. With the exception of PSD 96h the levels of IL-6 were significantly higher 175 than in controls in all PSD groups, including those measured after 192h PSD+R $[\mathrm{F}(4,25)=17.39$, $176 \mathrm{p}<0.0001]$ (Fig. 2A). PSD did not modify TNF concentrations as compared to controls and a 177 significant increment was only seen after recovery, at $192 \mathrm{~h} \mathrm{PSD}+\mathrm{R},[\mathrm{F}(4,25)=8.717, \mathrm{p}=0.0003]$

178 (Fig. 2B). IL-10 levels were significantly lower than in controls at PSD 24, raised significantly 179 after PSD 192h and dropped again below controls levels after PSD+R [F (4,25)=32.84, $\mathrm{p}<0.0001]$ 
180 (Fig. 2C). Finally, circulating levels of adiponectin showed a significant increase only after PSD $18196 \mathrm{~h}[\mathrm{~F}(4,25)=4.741, \mathrm{p}=0.0074]$ (Fig. 2D).

182 Changes in miRNA levels were found after the different periods of PSD and recovery

183 Serum levels of miRNA related to the inflammatory response were measured at the different 184 periods of PSD and after recovery. At PSD 24h three miRNA were found significantly increased 185 compared to controls: miR-146a $[\mathrm{F}(4,25)=9.538, \mathrm{p}=0.0002]$ (Fig. 3A), miR-155 [F $(4,25)=5.895$, $186 \mathrm{p}=0.0027]$ (Fig. 3B), and miR-223[F $(4,25)=21.75, \mathrm{p}<0.0001]$. The latter was observed decreased 187 at PSD 96h (Fig. 3C), as well as miR-16, which was found also decreased at PSD 192h and 188 PSD192+R compared to controls $[\mathrm{F}(4,25)=23.29$, $\mathrm{p}<0.0001]$ (Fig. 3D). miR-126 was decreased 189 at PSD 192h $[\mathrm{F}(4,25)=3.669, \mathrm{p}=0.0213]$ (Fig. 3E), and miR-21, showed no significant changes 190 during PSD, but an increase was observed at 192h PSD+R compared to controls and to PSD 192h $191[\mathrm{~F}(4,25)=15.48, \mathrm{p}<0.0001]($ Fig. 3F)

192

193 Discussion

194 We found that different periods of PSD modify corticosterone, IL-6, IL-10, and adiponectin levels, 195 depending on the duration of PSD. Most of these alterations in cytokines did not return to basal 196 values even after 20 days of recovery: only adiponectin did. TNF, which was not modified during 197 the different PSD periods, was found elevated only after recovery. Related to miRNAs, except for 198 miR-21 which was increased only during recovery, the levels of all the other inflammation related miRNAs (miR-146a, miR-155, miR-223, miR16, miR-126) were also modified by PSD. Notably, differentiating the miRNAs response to that of the cytokines, all these miRNAs but miR-16 which persisted in decreased levels, had returned to base-line levels after the recovery period. These findings suggest that PSD induces a dynamic response pattern that reflects cellular stress that is manifested as hormonal and inflammatory responses in different stages, that could be long-lasting.

204 REM sleep deprivation may induce a stress response (McEwen, 2006) that involves not only 205 behavioral responses, but is also associated with major modifications in brain biochemistry and in 206 the endocrine and immune systems, that may be dependent on the duration of REM sleep 207 deprivation in a response related to acute or chronic stress. After PSD 24h, elevated levels of the 208 inflammation related molecules IL-6 and miR-146a, miR-155 and miR-223 along with a decrease 
209 in the levels ofhe anti-inflammatory cytokine IL-10 were observed. Increased expression of miR-

210 146a has been previously reported in the cerebral cortex of rats that were sleep-deprived for $24 \mathrm{~h}$

211 (Matos et al., 2014). Particularly, since miR-146a is upregulated as NF-kB is activated and controls

212 cytokine signaling (Taganov, Boldin, Chang, \& Baltimore, 2006), this increase suggests that a

213 regulatory response is induced by short-term PSD (that is further lost after longer periods of PSD).

214 Also, it seems that the increase of these miRNAs at $24 \mathrm{~h}$ may be part of a rapid response to oxidative

215 stress and endotoxemia induced by sleep deprivation (Hirotsu, Matos, Tufik, \& Andersen, 2013)

216 processes that foster the secretion of proinflammatory cytokines, as in the case of IL-6 (Matos et

217 al., 2014 ; )Novotny et al., 2008). Furthermore, during acute stress a polymorphic region related

218 to the gene of the 5HT transporter modulates the response of inflammatory cytokines (Yamakawa,

219 Matsunaga, Isowa, \& Ohira, 2015).

220 During the "resistance phase" of stress, organisms manifest a process of adaptation to those 221 demands during a defined time, and activate a series of mechanisms in a stress response that aims 222 to restore homeostasis (Goldstein \& Kopin, 2007) which is what apparently happens when PSD223 induced stress is prolonged. During 96h of PSD, only miR-223, miR-16 and adiponectin were 224 modified, where the level of miR-223 and miR-16 decreased, but adiponectin increased. These 225 changes may be due to a compensatory response that seeks to limit tissue damage or dysfunction 226 related to both the metabolic and inflammatory alterations involved in this phenomenon 227 (Villarreal-Molina \& Antuna-Puente, 2012).

228 After a chronic sleep restriction (192h) we found increased serum levels of corticosterone, IL-6, 229 and IL-10. The stress and anti-inflammatory cytokines responses seem to be at their peaks. An 230 increase in the levels of IL-10 after long-term sleep deprivation (18h/day for 21 days) has been 231 previously reported (Venancio \& Suchecki, 2015). The increase in IL-6 after PSD 192h may be 232 part of this anti-inflammatory responses, since IL-6 has been described to play both pro233 inflammatory and anti-inflammatory roles because in some scenarios it stimulates IL-10 synthesis 234 (Petersen \& Pedersen, 2006), and inhibits TNF and IL-1 production (Wolkow et al., 2015). At this 235 point of PSD it seems that an effort to closely regulate inflammation is in process in order to control 236 its negative effects and the impairments associated with it in terms of performance, cognition, 237 sleepiness (Yirmiya \& Goshen, 2011) and the incidence of degenerative disorders such as diabetes 238 (Petersen \& Pedersen, 2006). 
239 Interestingly, at this point miRNAs seem not to be participating in an active control of

240 inflammation, but rather may reflect an exhaustion situation due to the long-term, sustained

241 wakefulness. At PSD 192h we found that serum miR-146a, miR-155, and miR-223 had returned

242 to base-line levels. However, low levels of miR-16 and miR-126 were observed. In other scenarios

243 -for example, under hyperglycemic conditions- these two miRNAs participate in the suppression

244 of inflammation and ROS production (Harris, Yamakuchi, Ferlito, Mendell, \& Lowenstein, 2008;

245 Tang, Wang, Shao, Wang, \& Zhu, 2017). Also, decreased serum expression of miR-126 (Chen et

246 al., 2016) and miR-16 in the brain (Li et al., 2010) has been associated with type II diabetes. Other

247 reports also indicate that miR-16 may play a role in the active coping mechanisms for stress

248 resilience (Zurawek et al., 2017). This miRNA is highly-expressed in serotoninergic (5-HT) and

249 noradrenergic neurons (NA) (Obrietan \& Hansen, 2013), both of which show high activity during

250 wakefulness, and enhance their functioning during sleep deprivation (Hipólide et al., 2005).

251

252 Reestablishing homeostatic processes is important for organisms, as it induces a period of 253 recuperation and repair after subjection to a stressful condition. It is interesting to note that even 254 after a 20-day recovery period the consequences of a chronic sleep deprivation were present as 255 alterations in biological mediators. Serum IL-10 and miR-16 were found decreased, while 256 corticosterone, IL-6, TNF, and miR-21 levels were increased in the 192h PSD+R group compared 257 to controls. A chronic sleep deprivation is undoubtedly a stressful situation. Thus, it is plausible 258 that one possible mediator of this delayed return to a homeostatic state would be corticosterone 259 since high levels were found after chronic PSD and even after a recovery period of 20 days. This 260 situation adds to the reported alteration in glucose metabolism (Brianza-Padilla et al., 2016) after 261 PSD. A previous study of the effects of stress hormones on SD showed that some miRNAs can be 262 regulated by corticosterone in tissue under experimental conditions (Mongrain et al., 2010).

263 Even when the reported recovery period was clearly not enough to a full-return to homeostasis, 264 there are hints to suggest the presence of a repairing response. For instance, since miR-21 has been 265 associated with the protection of various organs from suffering lesions (Tong, Xiao, Zeng, \& 266 Xiong, 2015, Xu et al., 2017), the elevated levels of miR-21 seen after the 20-day recovery period 267 posterior to $192 \mathrm{~h}$ of PSD may well be reflecting a mechanism aimed to restore functionality. 


\section{Conclusion}

269 Sleep deprivation may disrupt cytokine and miRNA production, seemingly related to stress and inflammatory responses. Changes in cytokines and miRNAs in sera seem to be related to the duration of sleep deprivation, as short-term PSD produced effects similar to those of an acute inflammatory response. Although an anti-inflammatory response may appear as PSD duration increases, the disruption of homeostasis probably leads to long-lasting variations of some set points that may be involved in the development and/or progression of several diseases.

\section{Acknowledgments}

276

277

278

279

280

281

282

283

284

285

286

287

288

289

290

291

292

293

294

295

We thank the Laboratorio Divisional de Biología Molecular de Ciencias Biológicas y de la Salud (UAM-I) for authorizing the use of the specialized equipment required for this study.

\section{References}

Adrien, J. (2002). Neurobiological bases for the relation between sleep and depression. Sleep Medicine Reviews, 6(5), 341-351.

Andersen, M. L., Martins, P. J. F., D’Almeida, V., Bignotto, M., \& Tufik, S. (2005).

Endocrinological and catecholaminergic alterations during sleep deprivation and recovery in male rats. Journal of Sleep Research, 14(1), 83-90. https://doi.org/10.1111/j.13652869.2004.00428.x

Brianza-Padilla, M., Bonilla-Jaime, H., Almanza-Pérez, J. C., López-López, A. L., SánchezMuñoz, F., \& Vázquez-Palacios, G. (2016). Effects of different periods of paradoxical sleep deprivation and sleep recovery on lipid and glucose metabolism and appetite hormones in rats. Applied Physiology, Nutrition, and Metabolism, 41(3), 235-243. https://doi.org/10.1139/apnm-2015-0337

Chen, J., Ning, R., Zacharek, A., Cui, C., Cui, X., Yan, T., ... Chopp, M. (2016). MiR-126 Contributes to Human Umbilical Cord Blood Cell-Induced Neurorestorative Effects After Stroke in Type-2 Diabetic Mice. STEM CELLS, 34(1), 102-113. https://doi.org/10.1002/stem.2193

Davis, C. J., Clinton, J. M., \& Krueger, J. M. (2012). MicroRNA 138, let-7b, and 125a inhibitors differentially alter sleep and EEG delta-wave activity in rats. Journal of Applied Physiology, 113(11), 1756-1762. https://doi.org/10.1152/japplphysiol.00940.2012 
297 Davis, C. J., Meighan, P. C., Taishi, P., Krueger, J. M., Harding, J. W., \& Wright, J. W. (2006).

298 REM sleep deprivation attenuates actin-binding protein cortactin: a link between sleep and 299 hippocampal plasticity. Neuroscience Letters, 400(3), 191-196.

$300 \quad \mathrm{https} / /$ doi.org/10.1016/j.neulet.2006.02.046

301 Dehoux, M. J. M., van Beneden, R. P., Fernández-Celemín, L., Lause, P. L., \& Thissen, J.-P. M. 302 (2003). Induction of MafBx and Murf ubiquitin ligase mRNAs in rat skeletal muscle after

Dietze-Schroeder, D., Sell, H., Uhlig, M., Koenen, M., \& Eckel, J. (2005). Autocrine action of adiponectin on human fat cells prevents the release of insulin resistance-inducing factors. Diabetes, 54(7), 2003-2011.

Goldstein, D. S., \& Kopin, I. J. (2007). Evolution of concepts of stress. Stress (Amsterdam, Netherlands), 10(2), 109-120. https://doi.org/10.1080/10253890701288935

Grandner, M., Sands-Lincoln, Pak, \& Garland. (2013). Sleep duration, cardiovascular disease, and proinflammatory biomarkers. Nature and Science of Sleep, 93. https://doi.org/10.2147/NSS.S31063

Haider, B. A., Baras, A. S., McCall, M. N., Hertel, J. A., Cornish, T. C., \& Halushka, M. K. (2014). A Critical Evaluation of microRNA Biomarkers in Non-Neoplastic Disease. PLoS ONE, 9(2), e89565. https://doi.org/10.1371/journal.pone.0089565

Harris, T. A., Yamakuchi, M., Ferlito, M., Mendell, J. T., \& Lowenstein, C. J. (2008). MicroRNA-126 regulates endothelial expression of vascular cell adhesion molecule 1. Proceedings of the National Academy of Sciences, 105(5), 1516-1521. https://doi.org/10.1073/pnas.0707493105

Hipólide, D. C., Moreira, K. M., Barlow, K. B. L., Wilson, A. A., Nobrega, J. N., \& Tufik, S. (2005). Distinct effects of sleep deprivation on binding to norepinephrine and serotonin transporters in rat brain. Progress in Neuro-Psychopharmacology and Biological Psychiatry, 29(2), 297-303. https://doi.org/10.1016/j.pnpbp.2004.11.015

Hirotsu, C., Matos, G., Tufik, S., \& Andersen, M. L. (2013). Changes in gene expression in the frontal cortex of rats with pilocarpine-induced status epilepticus after sleep deprivation. Epilepsy \& Behavior, 27(2), 378-384. https://doi.org/10.1016/j.yebeh.2013.02.024

Holm, A., Bang-Berthelsen, C. H., Knudsen, S., Modvig, S., Kornum, B. R., Gammeltoft, S., \& Jennum, P. J. (2014). miRNA profiles in cerebrospinal fluid from patients with central 
328

329

330

331

332

333

334

335

336

337

338

339

340

341

342

343

344

345

346

347

348

349

350

351

352

353

354

355

356

357

358

hypersomnias. Journal of the Neurological Sciences, 347(1-2), 199-204.

https://doi.org/10.1016/j.jns.2014.09.047

Irwin, M. R. (2006). Sleep Deprivation and Activation of Morning Levels of Cellular and Genomic Markers of Inflammation. Archives of Internal Medicine, 166(16), 1756. https://doi.org/10.1001/archinte.166.16.1756

Jones, B. E. (1998). The neural basis of consciousness across the sleep-waking cycle. Advances in Neurology, 77, 75-94.

Krueger, J. M., Frank, M. G., Wisor, J. P., \& Roy, S. (2016). Sleep function: Toward elucidating an enigma. Sleep Medicine Reviews, 28, 46-54. https://doi.org/10.1016/j.smrv.2015.08.005

Krueger, J. M., Obál, F. J., Fang, J., Kubota, T., \& Taishi, P. (2001). The role of cytokines in physiological sleep regulation. Annals of the New York Academy of Sciences, 933, 211-221.

Li, K., Wei, P., Qin, Y., \& Wei, Y. (2017). MicroRNA expression profiling and bioinformatics analysis of dysregulated microRNAs in obstructive sleep apnea patients. Medicine, 96(34), e7917. https://doi.org/10.1097/MD.0000000000007917

Li, T., Morgan, M. J., Choksi, S., Zhang, Y., Kim, Y.-S., \& Liu, Z. (2010). MicroRNAs modulate the noncanonical transcription factor NF- $\kappa \mathrm{B}$ pathway by regulating expression of the kinase IKK $\alpha$ during macrophage differentiation. Nature Immunology, 11(9), 799-805. https://doi.org/10.1038/ni.1918

Lorton, D., Lubahn, C. L., Estus, C., Millar, B. A., Carter, J. L., Wood, C. A., \& Bellinger, D. L. (2006). Bidirectional Communication between the Brain and the Immune System: Implications for Physiological Sleep and Disorders with Disrupted Sleep. Neuroimmunomodulation, 13(5-6), 357-374. https://doi.org/10.1159/000104864

Machado, R. B., Hipólide, D. C., Benedito-Silva, A. A., \& Tufik, S. (2004). Sleep deprivation induced by the modified multiple platform technique: quantification of sleep loss and recovery. Brain Research, 1004(1-2), 45-51. https://doi.org/10.1016/j.brainres.2004.01.019

Machado, R. B., Tufik, S., \& Suchecki, D. (2010). Modulation of Sleep Homeostasis by Corticotropin Releasing Hormone in REM Sleep-Deprived Rats. International Journal of Endocrinology, 2010, 1-12. https://doi.org/10.1155/2010/326151

Matos, G., Scorza, F. A., Mazzotti, D. R., Guindalini, C., Cavalheiro, E. A., Tufik, S., \& Andersen, M. L. (2014). The effects of sleep deprivation on microRNA expression in rats submitted to pilocarpine-induced status epilepticus. Progress in Neuro- 
359

360

361

362

363

364

365

366

367

368

369

370

371

372

373

374

375

376

377

378

379

380

381

382

383

384

385

386

387

388

389

Psychopharmacology and Biological Psychiatry, 51, 159-165. https://doi.org/10.1016/j.pnpbp.2014.02.001

McEwen, B. S. (2006). Sleep deprivation as a neurobiologic and physiologic stressor: allostasis and allostatic load. Metabolism, 55, S20-S23. https://doi.org/10.1016/j.metabol.2006.07.008

Mongrain, V., Hernandez, S. A., Pradervand, S., Dorsaz, S., Curie, T., Hagiwara, G., ... Franken, P. (2010). Separating the contribution of glucocorticoids and wakefulness to the molecular and electrophysiological correlates of sleep homeostasis. Sleep, 33(9), 1147-1157.

Novotny, N. M., Grosfeld, J. L., Turner, K. E., Rescorla, F. J., Pu, X., Klaunig, J. E., ... Sandoval, J. A. (2008). Oxidative status in neuroblastoma: a source of stress? Journal of Pediatric Surgery, 43(2), 330-334. https://doi.org/10.1016/j.jpedsurg.2007.10.040

Obrietan, K., \& Hansen, K. (2013). MicroRNA as therapeutic targets for treatment of depression. Neuropsychiatric Disease and Treatment, 1011. https://doi.org/10.2147/NDT.S34811

Peñalva, R. G., Lancel, M., Flachskamm, C., Reul, J. M. H. M., Holsboer, F., \& Linthorst, A. C. E. (2003). Effect of sleep and sleep deprivation on serotonergic neurotransmission in the hippocampus: a combined in vivo microdialysis/EEG study in rats. The European Journal of Neuroscience, 17(9), 1896-1906.

Petersen, A. M. W., \& Pedersen, B. K. (2006). The role of IL-6 in mediating the antiinflammatory effects of exercise. Journal of Physiology and Pharmacology: An Official Journal of the Polish Physiological Society, 57 Suppl 1, 43-51.

Sahu, S., Kauser, H., Ray, K., Kishore, K., Kumar, S., \& Panjwani, U. (2013). Caffeine and modafinil promote adult neuronal cell proliferation during $48 \mathrm{~h}$ of total sleep deprivation in rat dentate gyrus. Experimental Neurology, 248, 470-481. https://doi.org/10.1016/j.expneurol.2013.07.021

Siegel, J. M. (2005). Clues to the functions of mammalian sleep. Nature, 437(7063), 1264-1271. https://doi.org/10.1038/nature04285

Suchecki, D., Tiba, P. A., \& Tufik, S. (2002). Paradoxical sleep deprivation facilitates subsequent corticosterone response to a mild stressor in rats. Neuroscience Letters, 320(12), 45-48.

Suchecki, D., \& Tufik, S. (2000). Social stability attenuates the stress in the modified multiple platform method for paradoxical sleep deprivation in the rat. Physiology \& Behavior, 68(3), 
390

391

392

393

394

395

396

397

398

399

400

401

402

403

404

405

406

407

408

409

410

411

412

413

414

415

416

417

418

419

420

309-316.

Taganov, K. D., Boldin, M. P., Chang, K.-J., \& Baltimore, D. (2006). NF- B-dependent induction of microRNA miR-146, an inhibitor targeted to signaling proteins of innate immune responses. Proceedings of the National Academy of Sciences, 103(33), 1248112486. https://doi.org/10.1073/pnas.0605298103

Tang, S., Wang, F., Shao, M., Wang, Y., \& Zhu, H. (2017). MicroRNA-126 suppresses inflammation in endothelial cells under hyperglycemic condition by targeting HMGB1. Vascular Pharmacology, 88, 48-55. https://doi.org/10.1016/j.vph.2016.12.002

Tobaldini, E., Costantino, G., Solbiati, M., Cogliati, C., Kara, T., Nobili, L., \& Montano, N. (2017). Sleep, sleep deprivation, autonomic nervous system and cardiovascular diseases. Neuroscience \& Biobehavioral Reviews, 74, 321-329. https://doi.org/10.1016/j.neubiorev.2016.07.004

Tong, B., Xiao, M.-Y., Zeng, J.-X., \& Xiong, W. (2015). MiRNA-21 promotes fibrosis in orbital fibroblasts from thyroid-associated ophthalmopathy. Molecular Vision, 21, 324-334.

Venancio, D. P., \& Suchecki, D. (2015). Prolonged REM sleep restriction induces metabolic syndrome-related changes: Mediation by pro-inflammatory cytokines. Brain, Behavior, and Immunity, 47, 109-117. https://doi.org/10.1016/j.bbi.2014.12.002

Vgontzas, A. N., Mastorakos, G., Bixler, E. O., Kales, A., Gold, P. W., \& Chrousos, G. P. (1999). Sleep deprivation effects on the activity of the hypothalamic-pituitary-adrenal and growth axes: potential clinical implications. Clinical Endocrinology, 51(2), 205-215.

Villarreal-Molina, M. T., \& Antuna-Puente, B. (2012). Adiponectin: Anti-inflammatory and cardioprotective effects. Biochimie, 94(10), 2143-2149. https://doi.org/10.1016/j.biochi.2012.06.030

Wolkow, A., Ferguson, S. A., Vincent, G. E., Larsen, B., Aisbett, B., \& Main, L. C. (2015). The Impact of Sleep Restriction and Simulated Physical Firefighting Work on Acute Inflammatory Stress Responses. PLOS ONE, 10(9), e 0138128. https://doi.org/10.1371/journal.pone.0138128

Xu, X., Song, N., Zhang, X., Jiao, X., Hu, J., Liang, M., ... Ding, X. (2017). Renal Protection Mediated by Hypoxia Inducible Factor-1 $\alpha$ Depends on Proangiogenesis Function of miR-21 by Targeting Thrombospondin 1. Transplantation, 101(8), 1811-1819. https://doi.org/10.1097/TP.0000000000001501 
421 Yamakawa, K., Matsunaga, M., Isowa, T., \& Ohira, H. (2015). Serotonin transporter gene

422 polymorphism modulates inflammatory cytokine responses during acute stress. Scientific 423 Reports, 5(1), 13852. https://doi.org/10.1038/srep13852

424 Yehuda, S., Sredni, B., Carasso, R. L., \& Kenigsbuch-Sredni, D. (2009). REM Sleep Deprivation 425 in Rats Results in Inflammation and Interleukin-17 Elevation. Journal of Interferon \& $426 \quad$ Cytokine Research, 29(7), 393-398. https://doi.org/10.1089/jir.2008.0080

427 Yirmiya, R., \& Goshen, I. (2011). Immune modulation of learning, memory, neural plasticity 428 and neurogenesis. Brain, Behavior, and Immunity, 25(2), 181-213.

429 https://doi.org/10.1016/j.bbi.2010.10.015

430 Zurawek, D., Kusmider, M., Faron-Gorecka, A., Gruca, P., Pabian, P., Solich, J., ... Dziedzicka431 Wasylewska, M. (2017). Reciprocal MicroRNA Expression in Mesocortical Circuit and Its 432 Interplay with Serotonin Transporter Define Resilient Rats in the Chronic Mild Stress. 433 Molecular Neurobiology, 54(8), 5741-5751. https://doi.org/10.1007/s12035-016-0107-9 434 435 436 437 438 439 440 441 442 443 444 
Figure 1

Serum corticosterone levels during PSD.

The corticosterone levels increases bye effect of PSD at 192h and 192h+R. Values represent means \pm SEM. ANOVA followed by Tukey's tests were performed $* p<0.05$ compared to control; $\varphi p<0.05$ compared to the PSD 192h+R group.

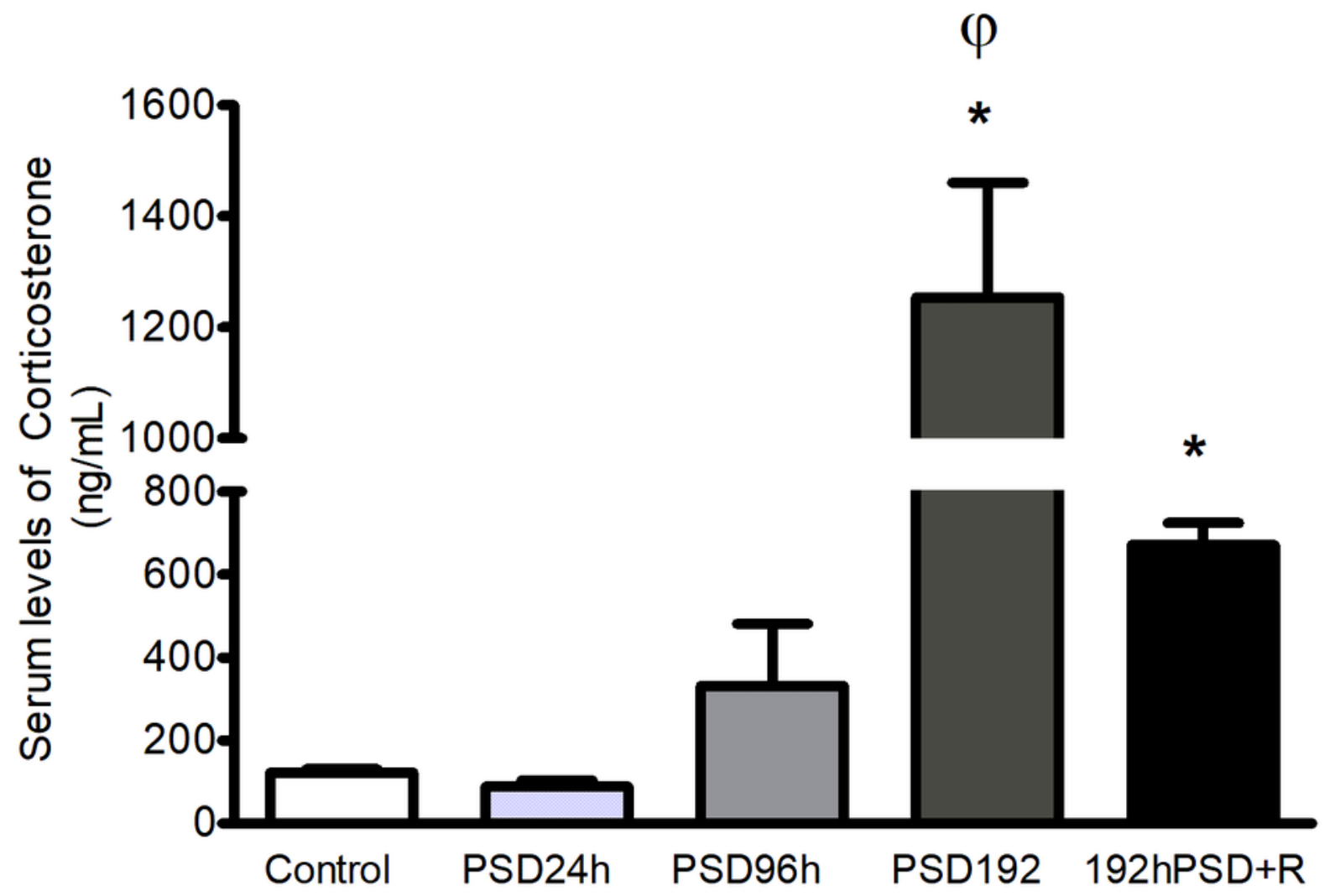


Figure 2

Effect of different periods of PSD on IL-6 (A), TNF (B), IL-10 (C), and adiponectin (D) concentrations

Values represent means \pm SEM. ANOVA followed by Tukey's tests were performed; ${ }^{*} p<0.05$ compared to the control group; $\varphi p<0.05$ compared to PSD $192 h+R .(n=5$ for each group).

A
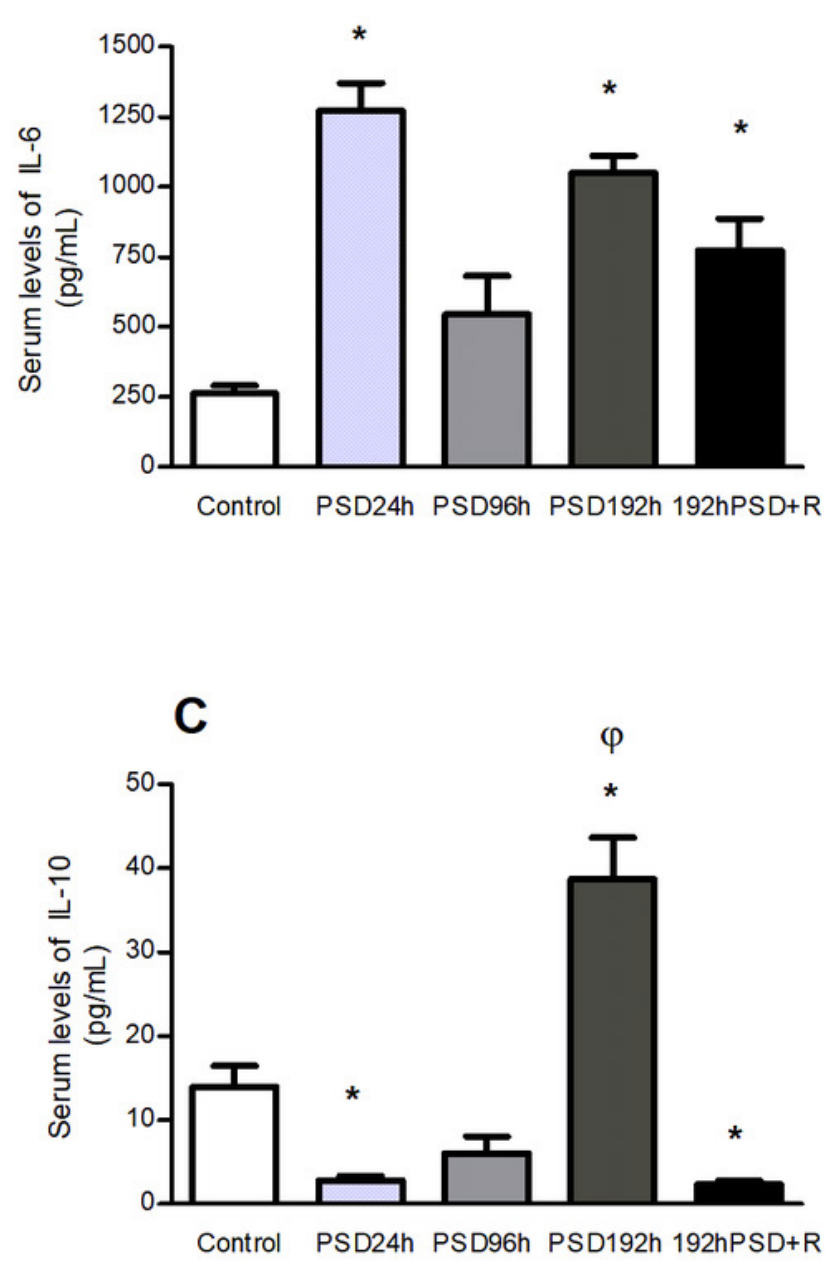

B
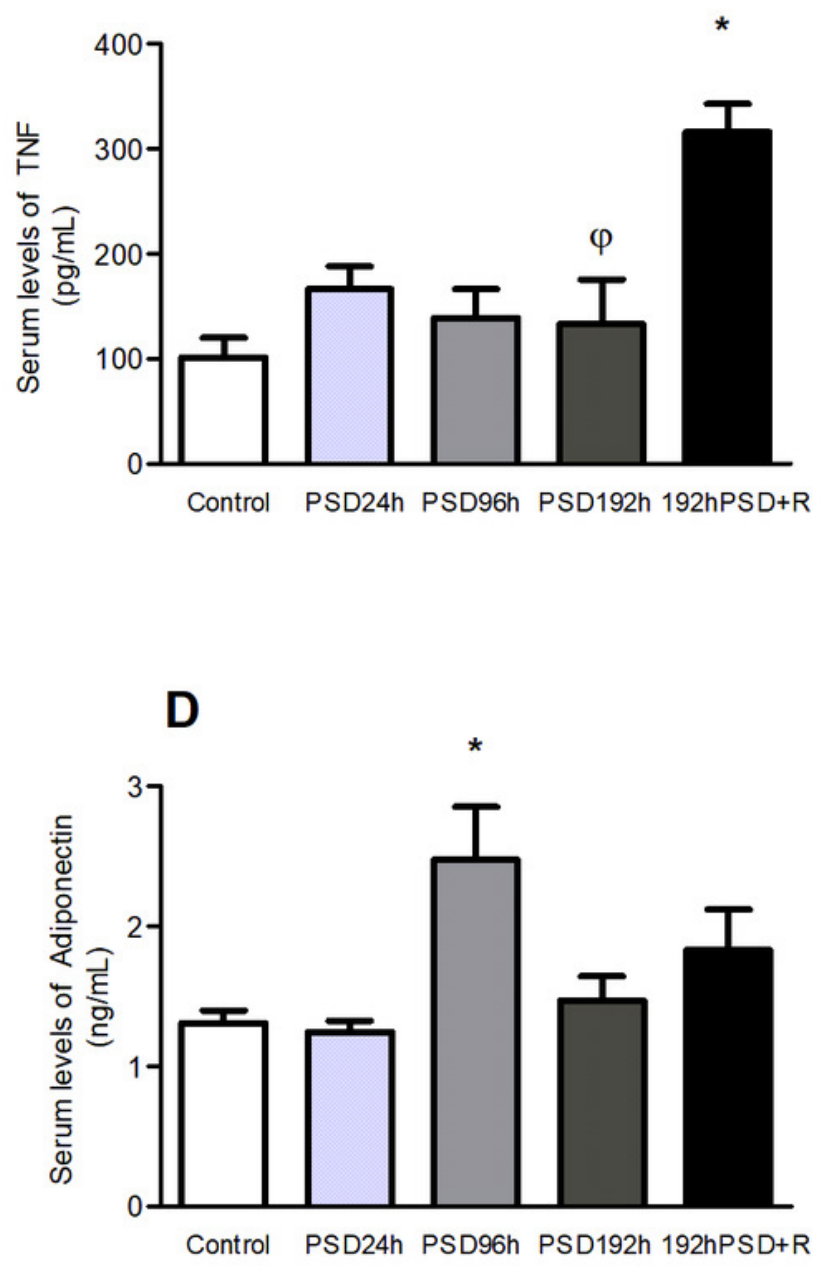


\section{Figure 3}

miRNAs levels in the sera of rats after different periods of PSD and recovery: miR-146a (A), miR-155 (B), miR-223 (C), miR-16 (D), miR-126 (E), and miR-21 (F)

Values represent means \pm SEM. An ANOVA followed by a Tukey's test was performed. ${ }^{*} p<0.05$ compared to the control group, and $\varphi p<0.05$ compared to the $192 \mathrm{~h}$ PSD+R group. 
A
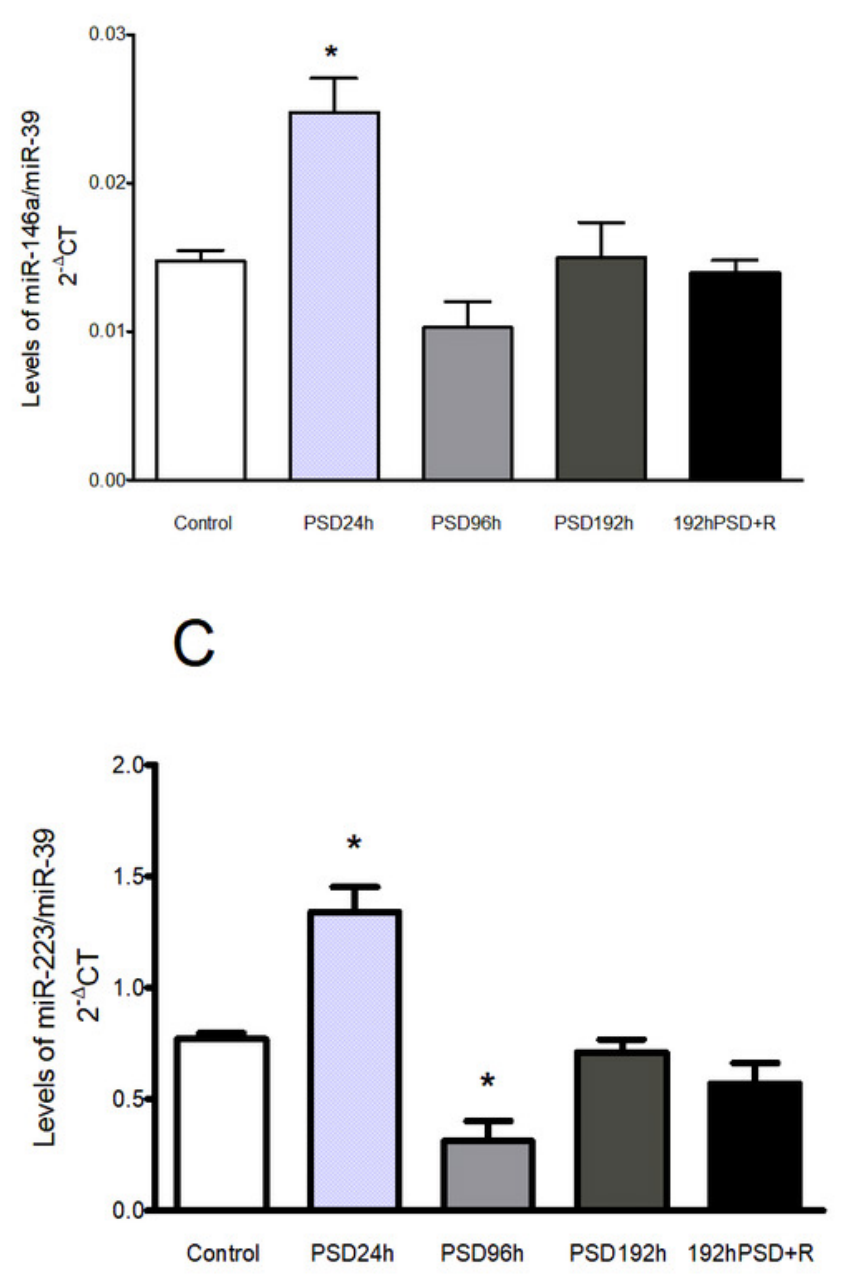

E

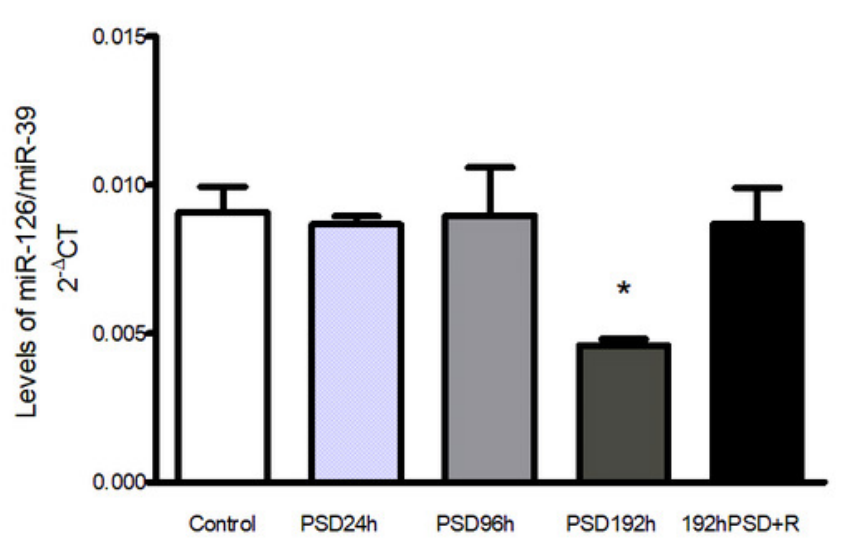

B
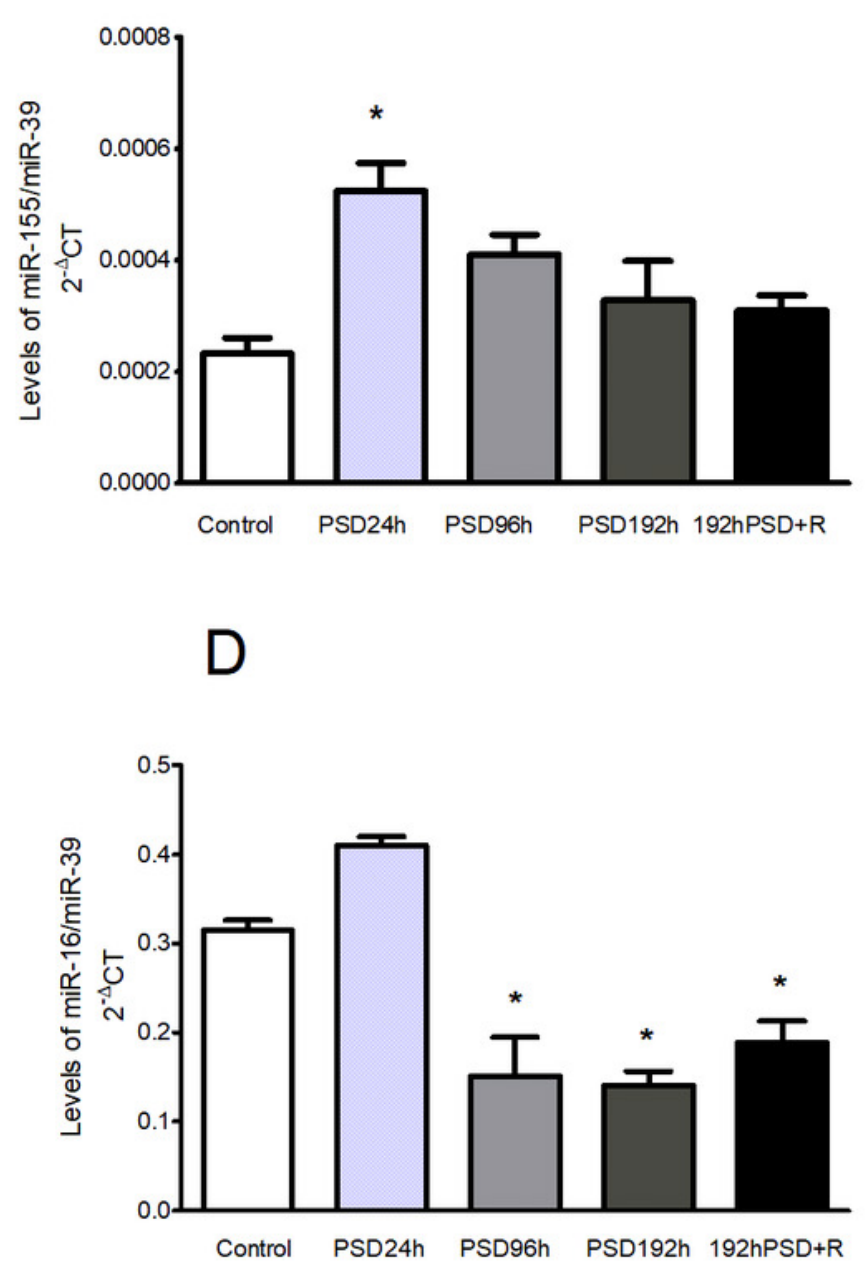

F

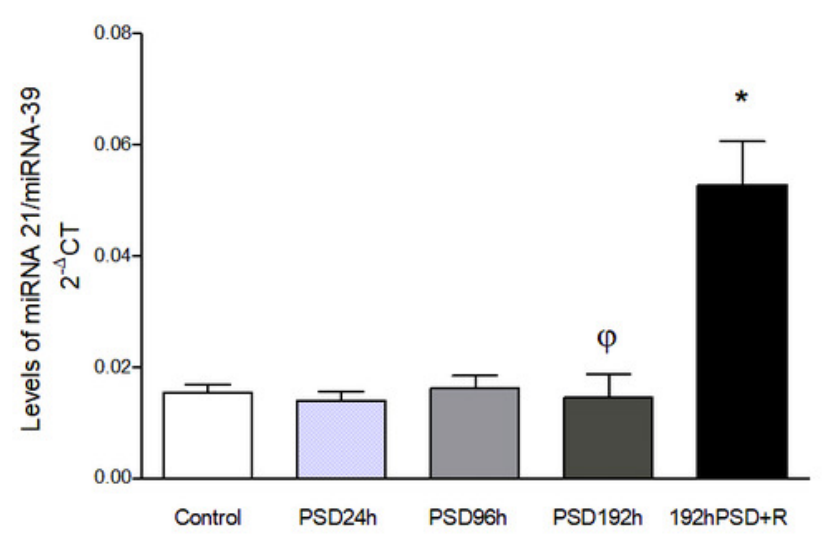

